\title{
C. Targeted fascicular biopsy of the brachial plexus: rationale and operative technique
}

\author{
Pierre Laumonerie, MD, ${ }^{1,2}$ Stepan Capek, MD, ${ }^{3}$ Kimberly K. Amrami, MD, ${ }^{4}$ P. James B. Dyck, MD, ${ }^{5}$ \\ and Robert J. Spinner, MD² \\ 'Department of Orthopaedic Surgery, Hôpital Pierre Paul Riquet, Toulouse, France; Departments of 2Neurologic Surgery, \\ ${ }^{4}$ Radiology, and ${ }^{5}$ Neurology, Mayo Clinic, Rochester, Minnesota; and ${ }^{3}$ Department of Neurological Surgery, University of Virginia, \\ Charlottesville, Virginia
}

OBJECTIVE Nerve biopsy is useful in the management of neuromuscular disorders and is commonly performed in distal, noncritical cutaneous nerves. In general, these procedures are diagnostic in only $20 \%-50 \%$. In selected cases in which preoperative evaluation points toward a more localized process, targeted biopsy would likely improve diagnostic yield. The authors report their experience with targeted fascicular biopsy of the brachial plexus and provide a description of the operative technique.

METHODS All cases of targeted biopsy of the brachial plexus biopsy performed between 2003 and 2015 were reviewed. Targeted nerve biopsy was performed using a supraclavicular, infraclavicular, or proximal medial arm approach. Demographic data and clinical presentation as well as the details of the procedure, adverse events (temporary or permanent), and final pathological findings were recorded.

RESULTS Brachial plexus biopsy was performed in 74 patients (47 women and 27 men). The patients' mean age was 57.7 years. All patients had abnormal findings on physical examination, electrodiagnostic studies, and MRI. The overall diagnostic yield of biopsy was $74.3 \%(n=55)$. The most common diagnoses included inflammatory demyelination (19), breast carcinoma (17), neurolymphomatosis (8), and perineurioma (7). There was a $19 \%$ complication rate; most of the complications were minor or transient, but 4 patients $(5.4 \%)$ had increased numbness and $3(4.0 \%)$ had additional weakness following biopsy.

CONCLUSIONS Targeted fascicular biopsy of the brachial plexus is an effective diagnostic procedure, and in highly selected cases should be considered as the initial procedure over nontargeted, distal cutaneous nerve biopsy. Using MRI to guide the location of a fascicular biopsy, the authors found this technique to produce a higher diagnostic yield than historical norms as well as providing justification for definitive treatment.

https://thejns.org/doi/abs/10.3171/2017.1.FOCUS16404

KEY WORDS brachial plexus; fascicular biopsy; surgery; technique

$\mathrm{N}$

ERVE biopsy is commonly employed for the diagnosis of neuromuscular disorders. Superficial radial and sural nerves are the 2 most common sites of biopsy. ${ }^{8,10,15,18}$ Distal cutaneous nerve biopsy allows a simple surgical approach. ${ }^{10}$ Typically, the sensory loss is minimal and acceptable without any functional deficit. While sacrifice of a cutaneous nerve can, in rare cases, result in a painful neuroma, the risk is acceptable. ${ }^{10}$ Biopsy of a cutaneous nerve has a moderately low diagnostic yield, ranging from $20 \%$ to $50 \%,{ }^{10}$ especially in motor neuropathies. ${ }^{17,18}$ Biopsy of a motor nerve is occasionally performed to distinguish between motor neuropathy and motor neuron disease.,12 In these cases, the obturator nerve branch to the gracilis muscle, the pronator branch of the median nerve, or the superficial peroneal nerve (more proximally) has been biopsied. ${ }^{1,2,7}$ However, biopsy of a motor nerve increases the risk of serious neurological sequelae, including weakness (and sensory loss in the case of mixed nerves). The balance of risks and benefits for this procedure must be considered.

We believe that we can improve the diagnostic yield of

ABBREVIATIONS CIDP = chronic inflammatory demyelinating polyneuropathy; IVIG = intravenous immunoglobulin; NCS/EMG = nerve conduction studies and electromyography; NOS = not otherwise specified; PET/CT = positron emission tomography-computed tomography.

SUBMITTED September 19, 2016. ACCEPTED January 4, 2017.

INCLUDE WHEN CITING DOI: 10.3171/2017.1.FOCUS16404. 
nerve biopsy in proximal or asymmetrical neuropathy by performing a targeted proximal biopsy. Targeting the most pathologically involved segment of the nerve based on meticulous preoperative planning may be considered to guide the choice of site of the biopsy and maximize its diagnostic accuracy. ${ }^{5}$ To a large extent this technique has been facilitated by a wide utilization of new high-resolution imaging techniques. ${ }^{3,5,11}$ Such guidance allows us to improve selectivity of a nerve biopsy site. ${ }^{4,5,9,19}$ Our group previously reported the experience of 112 targeted fascicular biopsies of the sciatic nerve in proximal lower-extremity neuropathies and lumbosacral plexopathies, showing this biopsy technique to be safe and efficacious in obtaining a histological diagnosis. ${ }^{5}$

In this report, we describe the results of our technique of fascicular biopsy applied to patients with brachial plexopathies and proximal neuropathies of the upper limb. As described above, a biopsy of the brachial plexus involves one to mixed motor/sensory nerve(s), with the distinct possibility of causing hyperesthesia, paresthesia, pain, or weakness. We balance these concerns with the knowledge that advances in nerve transfer surgery have shown that in some cases, even a functional nerve, branch, or fascicle can be sacrificed (and used for another function) with little, if any, donor morbidity. ${ }^{16}$ We are unaware of any other case series describing this technique in this location.

\section{Methods}

All cases of brachial plexus biopsies performed from 2003 through 2015 at the Mayo Clinic (Rochester, Minnesota) were reviewed after securing institutional review board approval. Targeted nerve biopsy was performed using a supraclavicular, infraclavicular, or proximal medial arm approach. Patients with mass lesions were excluded. All patients were examined by a neurologist (usually P.J.B.D.) and a single neurosurgeon (R.J.S.), both specializing in disorders of peripheral nerves. A radiologist with experience in peripheral nerve imaging (K.K.A.) also reviewed the imaging.

Demographic data (age and sex), clinical presentation (motor and sensory deficits, pain, laterality, and distribution of symptoms), and Tinel's sign were recorded for each patient. All results and interpretations of electrodiagnostic tests and imaging modalities, including MRI and positron emission tomography-computed tomography (PET/CT) were reviewed. Previous nerve, skin, and muscle biopsies were noted. Information from each targeted fascicular biopsy was collected, including the approach; biopsy sites; number, length, and appearance of fascicles taken; and function as elicited by muscle contraction in response to intraoperative electrical stimulation based on operative reports. Frozen and permanent section results were reviewed to report the diagnostic yield of this method. Treatment implications and postoperative complications of the targeted nerve biopsy were recorded.

\section{Evaluation}

Fascicles of the brachial plexus were selected for biopsy after careful evaluation of clinical presentation (history and physical examination) and additional tests (elec- trodiagnostic studies and imaging). Clinical examination provided an initial impression regarding localization of the disease. The point of maximum percussion tenderness (Tinel's sign) was used as an important sign because this has been well linked to the location of pathology. Nerve conduction studies and electromyography (NCS/EMG) further localized the disease and determined the severity. High-resolution 3-T MRI with T1-weighted, T2-weighted, or gradient echo-based sequences (e.g., spoiled gradient recalled echo) with very robust fat suppression, with and without gadolinium enhancement, was included as part of our standardized peripheral nerve imaging ${ }^{9-13}$ evaluation, demonstrating the presence and pattern of abnormalities in affected nerve(s) and fascicle(s). PET/CT was included in the preoperative evaluation of selected patients in whom malignancy was considered based on clinicoradiological features; increased avidity on PET would heighten suspicion for a malignant lesion as a justification for biopsy.

Nerve biopsy specimens were all taken to the peripheral nerve pathology laboratory, where histological preparations were made according to the laboratory protocol with teased fiber preparations and paraffin (longitudinal sections and cross-sections) and epoxy sections being made and standard stains being used. ${ }^{19}$ Special immunohistochemical preparations were performed on an as-needed basis.

\section{Operative Technique}

Once a specific target (nerve and location) was determined, a biopsy was planned. General anesthesia was used, and long-acting nondepolarizing neuromuscular blockers were avoided. Three surgical approaches were used according to the level of the biopsies: supraclavicular, deltopectoral, and proximal medial arm.

The supraclavicular approach was performed with a transverse incision of several centimeters (an average of 4.8 $\mathrm{cm})$ above the clavicle in one of Langer's lines, with preservation of supraclavicular nerves. After mobilization of the external jugular vein and omohyoid muscle, the anterior border of the anterior scalene muscle was identified along with the phrenic nerve. The spinal nerves, trunks, and divisions were then exposed and dissected as indicated.

The infraclavicular approach consisted of an incision with an average length of $8.3 \mathrm{~cm}$ in the deltopectoral interval. The cephalic vein was preserved and retracted. The pectoralis minor was taken down off its origin at the coracoid process, tagged and retracted. The axillary artery was then identified and mobilized. The neural elements of the brachial plexus (cords and branches) were exposed at this level.

The approach to the terminal branches of the brachial plexus was also performed by means of a longitudinal skin incision in the proximal arm measuring $7.6 \mathrm{~cm}$ on average and typically extending from the anterior axillary line distally in the medial arm.

The targeted nerve was exposed and inspected. If a focal abnormality was detected, it was biopsied. In the case where no striking abnormality was visualized, the biopsy was performed based solely on the abnormal findings on MRI. A nerve stimulator (Vari-Stim III nerve locator, Medtronic Xomed) helped identify nonfunctioning 
fascicles and map functioning fascicles. Typically, 1 or 2 fascicular groups that were stimulated without resulting in muscle contraction, preferably fascicular groups with a pathological appearance, were selected. In some cases when pathological findings were not apparent intraoperatively, 2 different areas in the same operative field were targeted to provide broad representative pathological samples with an acceptable risk of a postoperative deficit. Internal neurolysis was performed under loupe magnification. Although the neural interdigitations limited biopsy length, a goal of a length of $5 \mathrm{~cm}$ for each fascicle was desired (similar to the length of many distal cutaneous nerve biopsy specimens obtained by surgeons as per the protocol in our peripheral nerve laboratory); the length of fascicles obtained in our biopsy procedures averaged $5.3 \mathrm{~cm}$. Additional material was occasionally provided by an antebrachial cutaneous nerve.

After fascicle resection, a suture was placed in the proximal end and a lead weight was attached to the distal end to preserve nerve length. The resected fascicles were immediately divided into 3 pieces, with the long proximal piece hung directly in the operating room in $2.5 \%$ gluteraldehyde in buffer for 5 minutes and then transferred to $4 \%$ paraformaldehyde in buffer at $10^{\circ} \mathrm{C}$ and $\mathrm{pH}$ 7.4. The long distal piece was hung in $2.5 \%$ gluteraldehyde in buffer, isotonic with plasma, at $10^{\circ} \mathrm{C}$ and $\mathrm{pH} \mathrm{7.4}$. The short middle piece was immersed into melting isopentane in a metal beaker suspended in liquid nitrogen and stored at $-80^{\circ} \mathrm{C}$. These nerve specimens were then taken to the peripheral nerve laboratory for further processing. If malignancy was suspected, a small specimen was sent for frozen section. In all nerve biopsies, the epineurial blood vessels were inspected for the possibility of vasculitis.

Once the biopsy was achieved, the wound was closed in anatomical layers. In general, the procedure took less than 1 hour. Patients were either discharged home the same day or the next morning. The management of patients was determined 1-2 weeks postoperatively when the pathological diagnosis was finalized. Long-term clinical follow-up was performed or overseen.

\section{Results}

\section{Series Characteristics}

Seventy-four patients (47 women and 27 men) were included in the study. Their mean age was 57.7 years (range 3-87 years); 69 patients were adults, and 5 patients were children. Symptoms were present in the left upper limb in 32 patients (43.2\%), in the right upper limb in 31 (41.9\%), in both upper limbs in 9 (12.2\%), in both the right upper and the right lower limb in $1(1.3 \%)$ patient, and in all limbs in $1(1.3 \%)$ patient. All patients had abnormalities on clinical examination and on additional studies (EMG and MRI). Clinical examination revealed weakness in 74 patients (100\%), sensory loss in 64 (86.4\%) (sensory loss could not be assessed in the youngest patient, who was excluded from the sensory loss statement), Tinel's sign in 62 $(83.7 \%)$. Electrodiagnostic studies were diagnostic of brachial plexopathy in 58 patients (78.4\%) (49 unilateral, 9 bilateral), mononeuropathy in $11(14.9 \%)$, polyradiculopathy in $4(5.4 \%)$, and multiple neuropathies in 1 patient $(1.3 \%)$. MRI abnormalities were noted in all cases; the specific
TABLE 1. Pathological findings

\begin{tabular}{lc}
\hline \multicolumn{1}{c}{ Description } & No. of Cases \\
\hline Nondiagnostic & 19 \\
\hline Breast carcinoma & 17 \\
\hline Neurolymphomatosis & 8 \\
\hline Inflammatory demyelination neuropathy & 19 \\
\hline Perineurioma & 7 \\
\hline Granulocytic sarcoma & 1 \\
\hline Radiation injury & 1 \\
\hline MPNST & 1 \\
\hline Ischemic injury & 1 \\
\hline
\end{tabular}

MPNST = malignant peripheral nerve sheath tumor.

findings are beyond the scope of this article. Thirty-eight patients with a suspicion of malignant process underwent a PET/CT, which demonstrated increased uptake in the brachial plexus in 29 cases $(76.3 \%)$. Previous peripheral nerve biopsy was performed in 2 patients $(2.7 \%)$, muscle biopsy in 1 patient (1.3\%), and skin biopsy in 1 patient $(1.3 \%)$. None of these biopsies were diagnostic.

\section{Biopsies}

Seventy-four patients underwent targeted fascicular biopsy of the brachial plexus. A supraclavicular approach was performed in 33 patients $(44.6 \%)$, the deltopectoral approach in $33(44.6 \%)$, and a proximal arm approach in $8(10.8 \%)$. The biopsies were at the level of the spinal nerve in 21 cases $(28.4 \%)$, trunk in 24 (32.4\%), cord in $22(29.7 \%)$, lateral cord contribution to the median nerve in $9(12.2 \%)$, and terminal branches in 23 (31.1\%) (i.e., median [ $\mathrm{n}=8]$, radial $[\mathrm{n}=9]$, ulnar [ $\mathrm{n}=4]$, and axillary $[\mathrm{n}=2]$ nerves). Visible abnormalities were detected intraoperatively in 59 (79.7\%) patients. On average, 2 fascicles were sampled (range 1-6) with an average length of 5.3 $\mathrm{cm}$ (range $2.5-10 \mathrm{~cm}$ ). Multiple sites were biopsied at the same time in 25 patients ( 2 sites in 24 patients and 3 sites in 1 patient). In addition to the targeted fascicular biopsy, 9 patients had biopsy of another derived nerve (i.e., medial antebrachial cutaneous nerve in 6 patients and the lateral pectoral nerve, the subclavius nerve, and the coracobrachialis nerve in 1 patient each). Two patients had a biopsy of a neighboring lymph node, and 1 patient had a biopsy of the palmaris longus tendon. In 12 patients, neurolysis was performed at the time of biopsy to release nerve elements from extensive scarring.

\section{Pathology and Treatment Implications}

Overall, the biopsy yielded the diagnosis in 55 patients (74.3\%). The most common pathological diagnoses from the brachial plexus biopsies were malignancies (in 27 patients [36.5\%]) and inflammatory neuropathy (in 19 [25.7\%]) (Table 1).

Diagnoses allowed targeted treatment (Table 2). Patients with breast cancer or neurolymphoma in the brachial plexus were referred to an oncologist to start hormonal therapy or chemotherapy and/or radiotherapy. Of the 41 patients with cancer and clinicoradiological features worrisome for metastatic disease via perineural spread of breast cancer 
TABLE 2. Targeted treatment

\begin{tabular}{|c|c|c|c|c|c|c|c|c|c|c|}
\hline Condition & HT & RT & Chemo & $\begin{array}{c}\text { RT w/ } \\
\text { Chemo or HT }\end{array}$ & IVIG & $\begin{array}{c}\text { IV } \\
\text { Steroid }\end{array}$ & $\begin{array}{l}\text { Cyclophosphamide } \\
\text { w/ IVIG }\end{array}$ & $\begin{array}{l}\text { Tendon } \\
\text { Transfer }\end{array}$ & Amputation & $\begin{array}{l}\text { No } \\
\text { Tx }\end{array}$ \\
\hline Breast carcinoma & 1 & 5 & 3 & 8 & & & & & & \\
\hline Neurolymphomatosis & & 2 & 6 & & & & & & & \\
\hline Inflammatory demyelination neuropathy & & & & & 11 & 7 & 1 & & & \\
\hline Perineurioma & & & & & & & & 4 & & 3 \\
\hline Granulocytic sarcoma & & & 1 & & & & & & & \\
\hline Radiation injury & & & & & & & & & & 1 \\
\hline MPNST & & & & & & & & & 1 & \\
\hline Ischemic injury & & & & & & 1 & & & & \\
\hline
\end{tabular}

or neurolymphomatosis, 25 had biopsies positive for cancer. The 1 patient with 6 fascicles biopsied from 3 sites had MRI results that were highly suspicious, if not convincing, for breast cancer, but intraoperatively the confirmatory diagnosis was only established on biopsy at the third site; permanent sections did not reveal breast cancer in the first and second locations. In 14 patients, the biopsy was negative for cancer, and 1 patient was considered to have an inflammatory lesion, and another was considered to have postradiation changes. There was a selection bias in that only patients with a worrisome history, physical examination findings, and radiological features underwent biopsy procedures. As a result, in all of the patients with negative biopsy results, we maintained a high suspicion of malignancy, and these patients were closely followed. Four of these patients eventually developed clinically apparent tumor recurrence. We believe that a false-negative result was obtained in many of these cases and appreciate the difficulty in establishing a diagnosis of cancer in small nerve samples.

All of the patients with perineuriomas were being followed clinically without medical treatment; 4 were offered tendon transfers. After a diagnosis of inflammatory demyelination, treatment with intravenous immunoglobulin (IVIG) or steroids was started; 1 patient with multifocal motor neuropathy received cyclophosphamide. Many of these patients responded favorably to the new medical regimens.

\section{Adverse Events}

In this series, 14 patients (19\%) had complications following the biopsy; most of these complications were transient or minor (Table 3). Seven patients $(9.5 \%)$ developed neuropathic pain, 3 (4.0\%) developed increased weakness, $4(5.4 \%)$ developed new numbness, $1(1.3 \%)$ developed increased lymphedema in the operated limb, 4 (5.4\%) developed a self-limiting collection (hematoma or seroma), and $2(2.7 \%)$ developed urinary retention, which resolved spontaneously.

\section{Illustrative Cases \\ Case 1}

This 53-year-old woman presented to our department with recurrent pain radiating into the left dorsal hand with associated weakness. Physical examination revealed weakness involving the elbow, wrist, and digital and thumb ex- tension and diminished sensation in the radial snuff box. Tinel's sign was present for the radial nerve in the axilla and proximal arm.

The NCS/EMG was suggestive of chronic radial neuropathy, and MRI showed an oblong fusiform lesion with enhancement involving the posterior cord and the radial nerve in the axilla (Fig. 1A and B).

A targeted fascicular biopsy of the enlarged radial nerve was performed in the distal part of the deltopectoral groove (Fig. 1C). This confirmed the diagnosis of a benign intraneural perineurioma (Fig. 1D-G). This lesion was not resected, and tendon transfers were offered to the patient as treatment.

\section{Case 2}

This 51-year-old woman presented to our department with bilateral progressive upper-extremity numbness and weakness. She had been evaluated multiple times at other facilities without receiving a specific diagnosis. She had been initially diagnosed as having progressive bilateral carpal tunnel syndrome but did not respond to left carpal tunnel release or subsequent treatments with IVIG, methylprednisolone, and plasma exchange.

Seven NCS/EMG studies had been performed, most of which were suggestive of a multifocal patchy demyelinating process affecting both arms. MRI studies of the brachial plexus showed abnormalities involving the entire brachial plexus bilaterally, primarily the cords and proximal right median and ulnar nerves and the left radial nerve (Fig. 2A and B).

A nondiagnostic open biopsy of the median nerve and the flexor carpi radialis muscle at the forearm level had been performed prior to her referral to our institution. A fascicular biopsy of the right median nerve was then performed via a deltopectoral approach that showed large in-

TABLE 3. Adverse events

\begin{tabular}{lc}
\hline \multicolumn{1}{c}{ Description } & No. of Cases \\
\hline Neuropathic pain & 7 \\
\hline Fluid collection & 4 \\
\hline Numbness & 4 \\
\hline Weakness & 3 \\
\hline Urinary retention & 2 \\
\hline Increased arm lymphedema & 1 \\
\hline
\end{tabular}



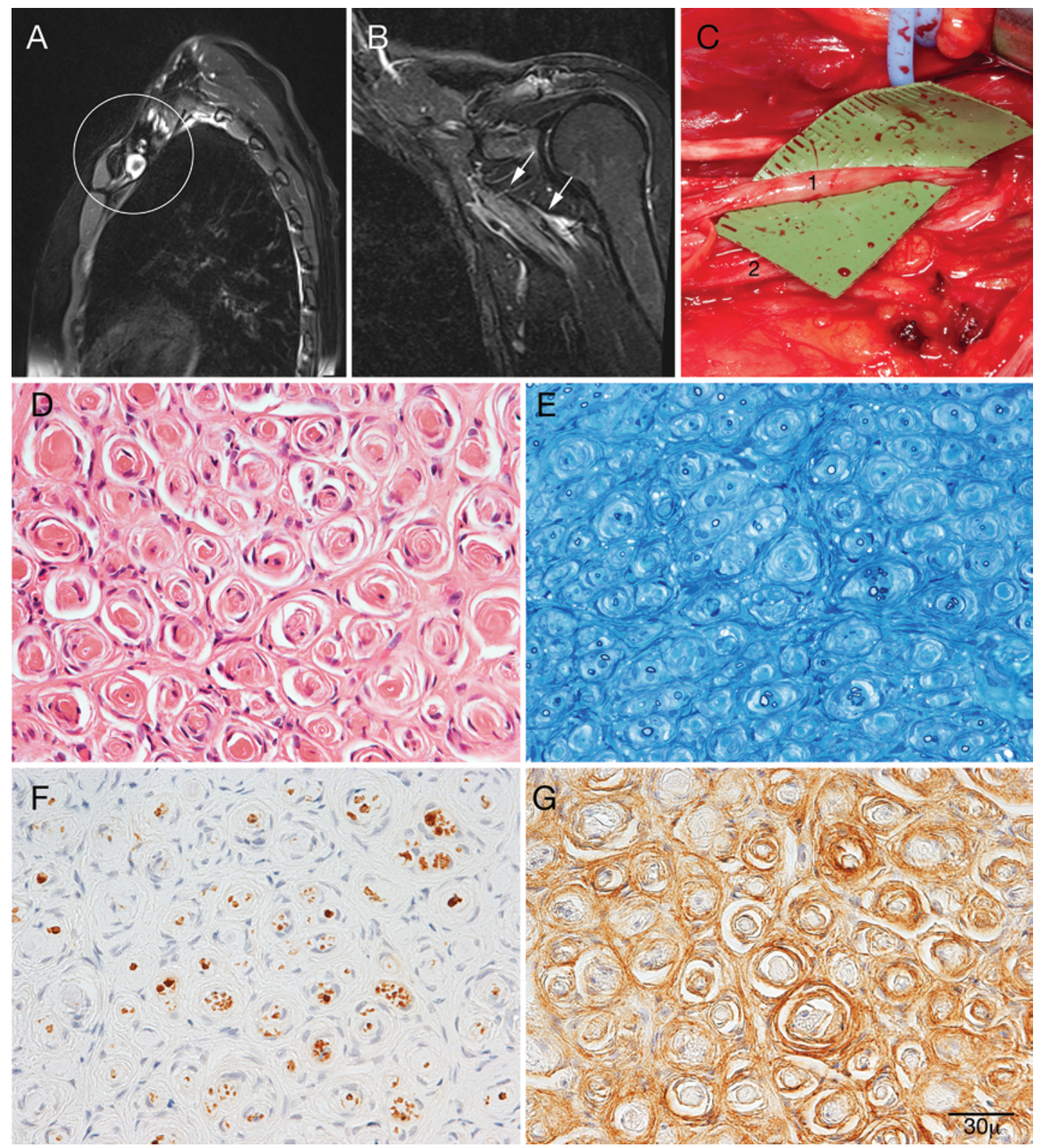

FIG. 1. Case 1. A and B: Sagittal (A) and coronal (B) fat-suppressed T2-weighted MR images of the left brachial plexus showing an elongated, fusiform hyperintense lesion involving the posterior cord (arrows), the radial nerve, and the axillary nerve in the axillary region. C: Operative photograph showing mobilization of a single fascicular group (1) from the medial portion of an enlarged radial nerve (2) through an infraclavicular exposure. D-G: Photomicrographs of serial paraffin cross-sections (D, F, and G) and epoxy section (E) from targeted nerve biopsy. $\mathrm{H}$ \& E staining (D) demonstrates diffuse pseudo-onion bulb formation. Epoxy section stained with methylene blue (E) demonstrates thinly myelinated fibers at the center of pseudo-onion bulbs. Schwann cell preparation (S-100, F) demonstrates reactivity of the myelinated fibers at the center and absence of reactivity of the surrounding pseudo-onion bulbs. Reactivity of pseudo-onion bulb leaflets with epithelial membrane antigen $(\mathrm{G})$ confirms that they are of perineurial origin. These findings taken together are diagnostic of perineurioma.

flammatory infiltrates in the endoneurium (Fig. 2C-E), and largely based on these findings the patient was diagnosed with chronic inflammatory demyelinating polyneuropathy (CIDP). No new deficit was recorded postoperatively, and an intravenous steroid regimen was started, resulting in significant improvement of her symptoms.

\section{Case 3}

This 54-year-old woman presented to our department with progressive numbness and paresthesias primarily involving the left little and ring finger associated with hand weakness and muscle atrophy and a focal tender spot without a true Tinel sign in the supraclavicular region over the lower trunk. In addition, the patient had a history of left breast cancer with metastatic disease (lung, liver, and bone), which had been treated with a bilateral mastectomy with adjuvant chemotherapy and radiotherapy.

The NCS/EMG results were suggestive of plexopathy, 

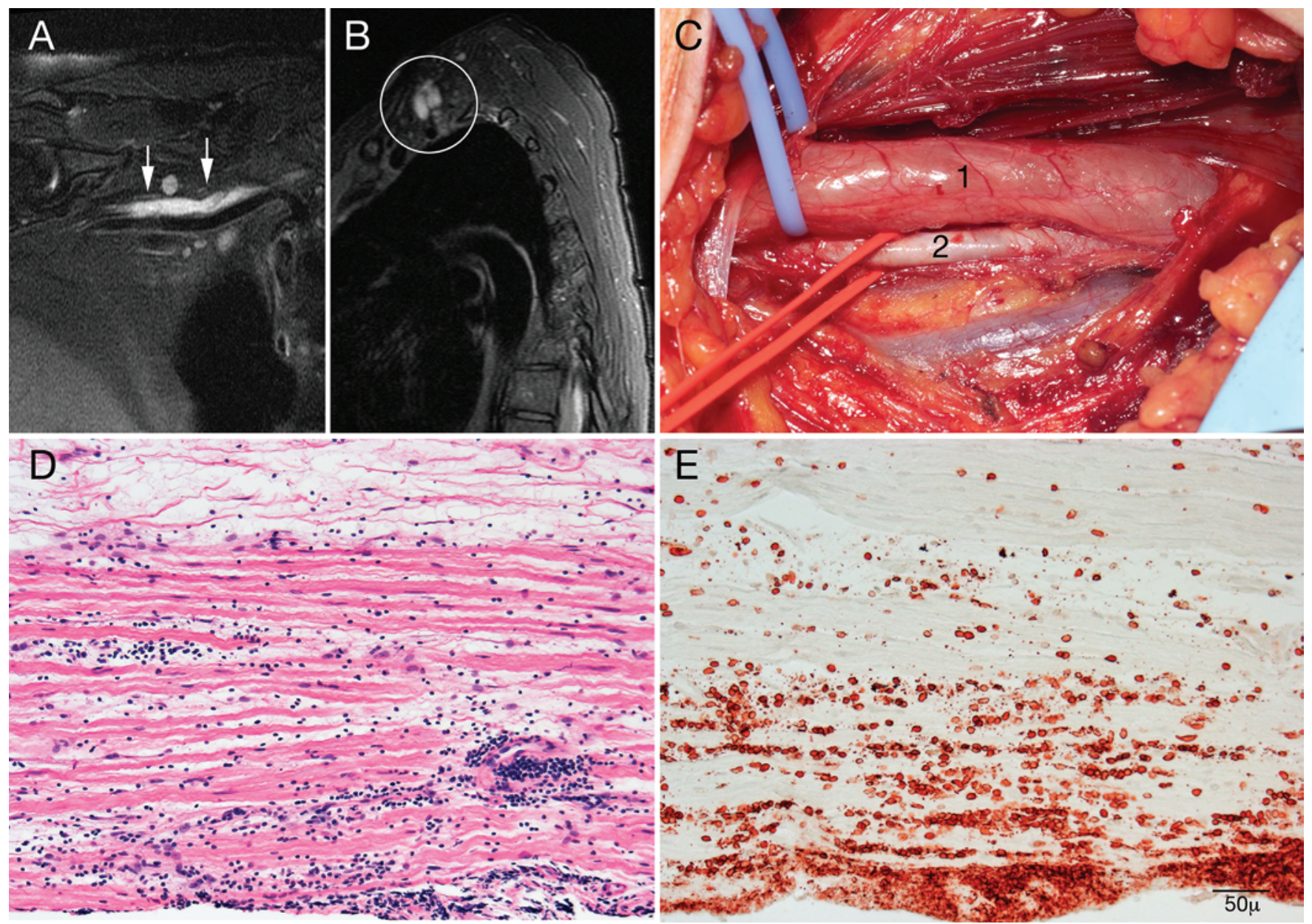

FIG. 2. Case 2. A and B: Oblique coronal (A) and sagittal (B) T2-weighted MR images of the right infraclavicular brachial plexus with fat suppression at the level of the cords showing enlargement and linear, fusiform hyperintensity in the brachial plexus (arrows in A, circle in B). C: Operative photograph showing marked enlargement of the right median nerve (1), approached using a deltopectoral approach. The axillary/brachial artery has also been mobilized in a red vasoloop (2). D and E: Longitudinal paraffin sections showing collections of perivascular endoneurial inflammatory cells (H \& E stain, D) and CD45 reactivity (common leukocyte antigen, E). Endoneurial inflammatory infiltrates are typical of inflammatory demyelination.

and MRI showed enlargement of the lower trunk of the left brachial plexus that was concerning for perineural tumor spread. PET/CT demonstrated significant uptake within the left brachial plexus without evidence for distant metastatic disease (Fig. 3A and B).

The patient underwent targeted fascicular biopsy of the enlarged lower trunk (Fig. 3C); histological examination of the biopsy specimen demonstrated infiltration with breast cancer. She had no new deficit after surgery and underwent chemotherapy with an excellent response: initially with relief of pain and later with some degree of recovery of hand function.

\section{Discussion}

In the present study, we showed that targeted fascicular biopsy of the brachial plexus has high diagnostic yield with an acceptable complication rate.

\section{Rationale and Indications}

Tissue Is the Issue

The main indications for targeted biopsy in our patient cohort included evaluation of an unusual clinical presentation without clear etiological findings or an evolution of focal neurological symptoms in the upper limb despite conservative management or concern for malignant processes, such as breast carcinoma and lymphoma.

Compared with historical results, $1,2,7,14,17$ our technique of targeted fascicular biopsy provides a more accurate diagnosis with histological confirmation, and it allows more rapid initiation of appropriate therapy compared with other techniques. However, the success of the procedure depends on careful patient selection and a dedicated team of neurologists, neurosurgeons, radiologists, and pathologists. Practitioners with a high level of expertise in peripheral nerve pathology are needed during every step of the process. Expert review of imaging studies and appropriate handling and interpretation of histological specimens are critical to ensure that the risk of the biopsy is offset by meaningful targeting of the biopsy and the highest level of neuropathological expertise for these complex cases. For example, most laboratories do not perform teased fiber preparation, which often requires specialized training but can help diagnose demyelinating lesions. 

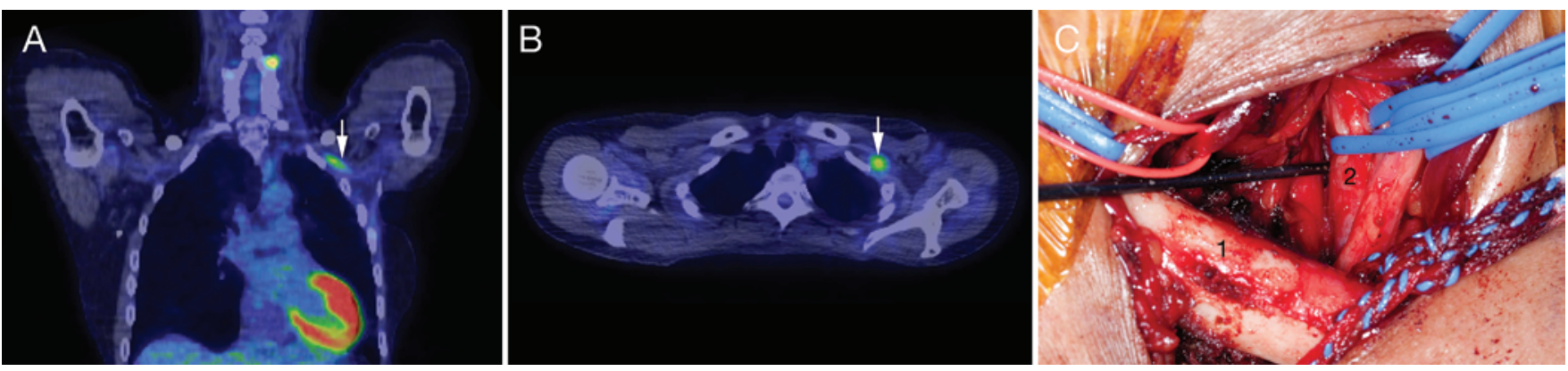

FIG. 3. Case 3. A and B: Coronal (A) and axial (B) FDG-PET/CT images showing linear uptake of FDG (arrows) consistent with malignant involvement of a portion of the left brachial plexus. C: Operative photograph showing the enlarged and discolored nonfunctioning lower trunk (2), accessed via a supraclavicular approach (1 indicates the clavicle).

\section{The Benefit Should Outweigh the Risk}

Benefit

In our study the diagnostic accuracy of a targeted fascicular biopsy was $74.3 \%$. Based on the current literature, this technique is more efficient than distal cutaneous nerve biopsy, ${ }^{10}$ especially for patients with focal brachial plexus pathology. The definitive diagnosis depends on accurate targeting of the pathological area of the brachial plexus. Notably, a higher failure rate $(25.7 \%)$ was observed in this study than was found in targeted fascicular biopsy of the sciatic nerve $(15.2 \%) .^{5}$ This can be potentially attributed to 1) the shorter length of the fascicles obtained due to the increased number of neural interconnections in the brachial plexus and 2) the lack of "functional overlap" in the brachial plexus compared with the sciatic nerve (particularly the tibial division). Patients received treatment based on the results of the biopsy. We believe that this targeted approach is better than an empirical one.

Many of the patients in this series had incorrect diagnoses prior to biopsy. For example, several of the patients diagnosed with intraneural perineuriomas had been previously treated medically for other possible causes of their neuropathy (i.e., with oral or intravenous steroids and/or IVIG) without clinical improvement prior to the evaluation at our medical facility. Since there is no known medical treatment for perineuriomas, empirical treatment that has medical risks and financial costs could be avoided.

\section{Risk}

Targeted fascicular biopsy of the brachial plexus is a relatively safe technique with a low complication rate. These results can be achieved by detailed knowledge of the complex anatomy of the brachial plexus and neighboring structures and careful intraoperative selection of nonfunctional (or noncritical) fascicles. In our experience, fascicular biopsy in selected patients is still worth the relatively small risk from surgery if one factors in the risks and costs of empirical treatment with intravenous steroids or IVIG in patients typically with a progressive neuropathy. However, the brachial plexus approach has to be performed with great caution, especially in the presence of extensive fibrosis and scarring. Patients who have undergone previous surgery and local radiation are at risk for wound-related problems, lymphedema, and neural complications. Appropriate patient preparation regarding the relative risks and benefits of the procedure is critical.

\section{Lessons Learned}

As a result of our long experience and multidisciplinary approach to peripheral nerve pathologies, we have learned some pearls for an efficient uncomplicated nerve biopsy. First, biopsies are not routinely performed for all abnormal nerves; several disorders, such as perineuriomas, lipomatosis of nerve, CIDP, and neuromuscular choristoma typically have pathognomonic radiological features based on our prior experience with and correlation of clinical, radiological, and histopathologic features., ${ }^{3,6,13,15}$ In addition, in some cases, we believe that nondiagnostic biopsies may have occurred when they were performed in radiological sites of abnormality that were distal to the actual pathology (so-called "downstream effect"); we do not know if obtaining a biopsy specimen "upstream" would be helpful. Quite simply, determining the exact target in patients with presumed neoplastic infiltration by breast cancer has been difficult.

\section{Future Directions}

The main goal of this procedure is to improve efficacy and safety. The technique and the results described can potentially be improved with higher-resolution imaging and better targeting of affected fascicles. Other imaging techniques, such as MR spectroscopy or diffusion tensor imaging could be helpful for more precise localization of pathology and improved detection/selection of an affected fascicle.

\section{Conclusions}

We present a series of 74 patients who underwent targeted fascicular biopsy of the brachial plexus, which yielded a diagnosis in $74.3 \%$ of cases. The diagnoses were very diverse, and on many occasions, the biopsy revealed a diagnosis allowing an appropriate therapy or a more aggressive therapy. We therefore believe that in select cases, targeted fascicular biopsy is an effective and appropriate diagnostic tool in managing patients with discrete and identifiable neurological lesions of the brachial plexus.

\section{Acknowledgments}

Dr. Laumonerie was supported by the French Society of Orthopedic Surgery and Traumatology (SOFCOT), Paris, France. 


\section{References}

1. Abouzahr MK, Lange DJ, Latov N, Olarte M, Rowland LP, Hays AP, et al: Diagnostic biopsy of the motor nerve to the gracilis muscle. Technical note. J Neurosurg 87:122-124, 1997

2. Agadi JB, Raghav G, Mahadevan A, Shankar SK: Usefulness of superficial peroneal nerve/peroneus brevis muscle biopsy in the diagnosis of vasculitic neuropathy. J Clin Neurosci 19:1392-1396, 2012

3. Amrami KK, Felmlee JP, Spinner RJ: MRI of peripheral nerves. Neurosurg Clin N Am 19:559-572, vi, 2008

4. Amrami KK, Spinner RJ, Dyck PJB, Felmlee JP: Techniques for magnetic resonance imaging of peripheral nerves: diagnosis and guidance for targeted fascicular nerve biopsy, in Dyck PJ, Amrami KK, Klein CJ, et al (eds): Companion to Peripheral Neuropathy: Illustrated Cases and New Developments. Philadelphia: Saunders Elsevier, 2010, pp 15-18

5. Capek S, Amrami KK, Dyck PJB, Spinner RJ: Targeted fascicular biopsy of the sciatic nerve and its major branches: rationale and operative technique. Neurosurg Focus 39(3):E12, 2015

6. Capek S, Hébert-Blouin MN, Puffer RC, Martinoli C, Frick MA, Amrami KK, et al: Tumefactive appearance of peripheral nerve involvement in hematologic malignancies: a new imaging association. Skeletal Radiol 44:1001-1009, 2015

7. Dy CJ, Lange DJ, Jones KJ, Garg R, DiCarlo EF, Wolfe SW: Diagnostic biopsy of the pronator teres and a motor branch of the median nerve: indications and technique. J Hand Surg Am 37:2570-2575, 2012

8. Dyck PJ, Lofgren EP: Nerve biopsy. Choice of nerve, method, symptoms, and usefulness. Med Clin North Am 52:885893, 1968

9. Dyck PJB, Spinner RJ, Amrami KK, Klein CJ, Engelstad JK, Dyck PJ: MRI-targeted fascicular nerve biopsies of proximal nerves: historic reports and illustrative case reports, in Dyck PJ, Amrami KK, Klein CJ, et al (eds): Companion to Peripheral Neuropathy: Illustrated Cases and New Developments. Philadelphia: Saunders Elsevier, 2010, pp 3-14

10. Hart MG, Santarius T, Trivedi RA: Muscle and nerve biopsy for the neurosurgical trainee. Br J Neurosurg 27:727-734, 2013

11. Howe BM, Spinner RJ, Felmlee JP, Amrami KK: High-resolution imaging of upper limb neuropathies. Semin Musculoskelet Radiol 19:160-167, 2015

12. Kennedy WR, Nolano M, Wendelschafer-Crabb G, Johnson TL, Tamura E: A skin blister method to study epidermal nerves in peripheral nerve disease. Muscle Nerve 22:360 371,1999

13. Mauermann ML, Amrami KK, Kuntz NL, Spinner RJ, Dyck PJ, Bosch EP, et al: Longitudinal study of intraneural peri- neurioma-a benign, focal hypertrophic neuropathy of youth Brain 132:2265-2276, 2009

14. Moss JP, Meckler RJ, Moss WE: Consistent, effective technique for muscle and nerve biopsy. Am J Surg 138:736-737, 1979

15. Niederhauser BD, Spinner RJ, Jentoft ME, Everist BM, Matsumoto JM, Amrami KK: Neuromuscular choristoma: characteristic magnetic resonance imaging findings and association with post-biopsy fibromatosis. Skeletal Radiol 42:567-577, 2013

16. Oberlin C, Durand S, Belheyar Z, Shafi M, David E, Asfazadourian $\mathrm{H}$ : Nerve transfers in brachial plexus palsies. Chir Main 28:1-9, 2009

17. Oh SJ: Diagnostic usefulness and limitations of the sural nerve biopsy. Yonsei Med J 31:1-26, 1990

18. Ruth A, Schulmeyer FJ, Roesch M, Woertgen C, Brawanski A: Diagnostic and therapeutic value due to suspected diagnosis, long-term complications, and indication for sural nerve biopsy. Clin Neurol Neurosurg 107:214-217, 2005

19. Spinner RJ, Amrami KK, Dyck PJB: Targeted fascicular biopsy: a surgical perspective, in Dyck PJ, Amrami KK, Klein CJ, et al (eds): Companion to Peripheral Neuropathy: Illustrated Cases and New Developments. Philadelphia: Saunders Elsevier, 2010, pp 19-24

\section{Disclosures}

The authors report no conflict of interest concerning the materials or methods used in this study or the findings specified in this paper.

\section{Author Contributions}

Conception and design: all authors. Acquisition of data: Spinner, Laumonerie, Capek. Analysis and interpretation of data: Spinner, Laumonerie, Capek, Dyck. Drafting the article: all authors. Critically revising the article: all authors. Reviewed submitted version of manuscript: all authors. Approved the final version of the manuscript on behalf of all authors: Spinner. Statistical analysis: Laumonerie. Administrative/technical/material support: Spinner. Study supervision: Spinner.

\section{Supplemental Information \\ Videos \\ Video Abstract. https://vimeo.com/204224378.}

\section{Correspondence}

Robert J. Spinner, Mayo Clinic, 200 1st St. SW, Gonda 8-214, Rochester, MN 55905. email: spinner.robert@mayo.edu. 